\author{
International Journal of Innovative Research in \\ Electrical, Electronics, Instrumentation and Control Engineering
}

Vol. 9, Issue 10, October 2021

DOI: 10.17148/IJIREEICE.2021.91015

\title{
An Overview of Bladeless Drone
}

\author{
Sivaraman $S^{1}$, Saravana Kumar $V^{2}$, Rajalakshmi $S^{3}$, Santhini $M^{4}$, Sittheshwaran $S^{5}$ \\ 1,2 Assistant Professor, Aeronautical Engineering, Hindusthan college of engineering and technology, \\ Coimbatore, Tamilnadu \\ ${ }^{3,4,5} \mathrm{UG}$ - Aeronautical Engineering, Hindusthan college of engineering and technology, Coimbatore, Tamilnadu
}

\begin{abstract}
The aim of the study project to design the blades drone by using bladeless technology. The motivation in this overview to eliminate the problems created by propellers on conventional multirotor. The bladeless propulsion system serve as alternative to propellers of drone The concept of bladeless drone inspired by dyson bladeless fan.
\end{abstract}

Keywords: Bladeless drone, bladeless technology, eliminate problems, dyson bladeless fan.

\section{INTRODUCTION}

The aim of the study project is to design a drone that uses impeller instead of propellers. The concept of propulsion system is another way of moving drones.

\section{METHODOLOGY}

\subsection{Disadvantages of propeller drone}

1. Small drones cannot cut your fingers, but if they fall on your body, they can damage the eyes.

2. Drug droplets can lead to deep cuts, splitting organs, and, in the worst case, even kill you if they cut you in one of your large arteries in the neck or wrists.

3. Drone shelters can be dangerous to birds and flying insects.

4. When a drone hits an object, the propeller is usually struck first and easily destroyed.

\subsection{Bladeless drone concepts}

1. Promoted from Dyson Bladeless Fans

2. It uses Closed Impeller to absorb air and distributes the loops powered by BLDC Motor.

3. Barriers use Inducement, Entrainment and Coanda Effect

4. It is up to 15 times more powerful than a fan of the same size.

5. The Bladeless Dragon was designed by Edgar Herrera, and exhibited in Yanko Design.

6. The technology behind this drone uses propellers, but they do not need drone aircraft belts.

7. This aerodynamic design is similar to the way modern aircraft are designed, but with a different body.

8. The main body is fitted with a large air duct that helps up and down.

9. The air flowing between the drone body pipes is used by a propeller. These parts are found in the inner holes of the plane.

10. When the drone is in the air, three small propellers are used to produce a low flow that keeps the wire going.

\section{COMPONENTS}

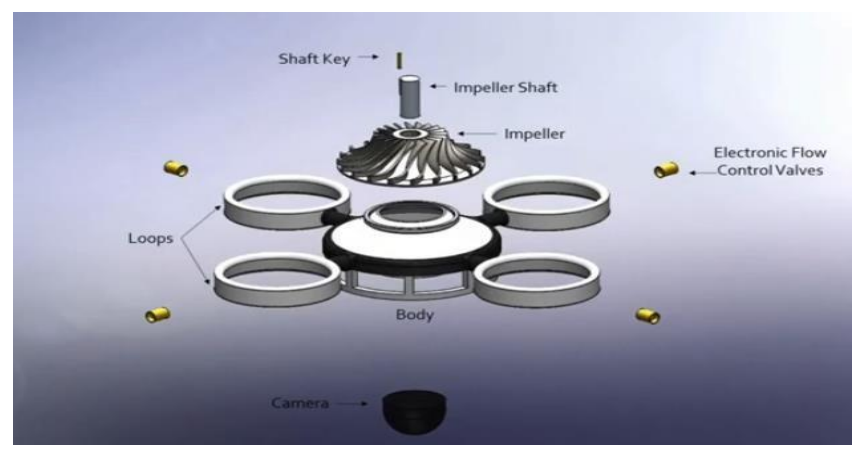

Fig. 1. Various parts 


\section{International Journal of Innovative Research in \\ Electrical, Electronics, Instrumentation and Control Engineering}

Vol. 9, Issue 10, October 2021

DOI: 10.17148/IJIREEICE.2021.91015

The main parts consist of

1. Battery

2. Dyson fan

3. Impeller

4. Impeller shaft

5. Electronic loop valves

\section{Battery}

Li-Po batteries are more energy-efficient and are commonly used for electric aircraft. They are also lighter than their predecessors. In addition, they require special charging cords and are more durable.

\section{Bladeless fan}

A bladeless fan, as the name suggests, has no visible blades and emits air from within a ring-like hollow.

There are two main types of bladeless fans: the Elongated-Oval and the Spherical-Oval. Both of these types have a hollow base and retractable blades.

This fan uses a multi-plier technology to create an impressive air flow in the room. Its base unit draws air in through a series of $16 \mathrm{~mm}$ slits, and the air is pushed out through the holes.

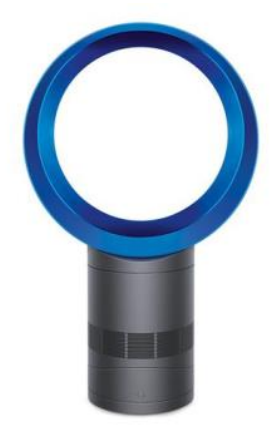

Fig. 2. Dyson fan

\section{Power consumption of a bladeless fan}

Bladeless fans work on a simple mechanism - to suck in air and push it out. Hence, they do not consume a large amount of electricity and are energy efficient. So, you won't have to worry about your mounting electricity costs.

Benefits of a bladeless fan over standard fans

An empty fan is a new trend in the fan industry. It has great strength as it repeats the outflow of air, therefore, it provides the best cooling. An bladeless follower has more advantages than regular fans.

1. Since it has no outer covers, it is much safer than a regular roof or table follower as there is no fear of cutting it.

2. An empty fan tube makes it easy to clean. While the average fan blades collect dust easily, the same is not true for an bladeless fan.

3. It can be controlled remotely. Therefore, you do not need to adjust the fan speed manually.

4. It is much easier than the average fan. Therefore, it can be easily moved from one place to another.

5. The cords of a normal fan start by breaking the air before they reach you, thus, causing the blow. No problem being beaten by an empty fan since you don't have belts.

6. It is interesting to look at. Sleek and elegant, these fans have a unique design that can blend well with the furniture in your room.

\section{How does a dyson follower work?}

The bladeless follower works on a very simple technology, as mentioned above, air-conditioning technology.

It consists of an electric motor without brushes and nine asymmetrically aligned valves attached to the Rotor. The frame is slightly held in the corner so that its bending can create additional pressure. As the air continues to flow, the surrounding air absorbs more quickly, or is "embedded" in the machine, creating a strong air flow. This is how an bladeless follower can make the air move. It is known to increase air flow by up to 15 times compared to the average fan. James Dyson was the man behind this idea of breaking the world.

\section{Breastfeeding}

An unmanned vehicle draws up to 25 gallons [27 L] of air per second. The technology used on the fan is similar to that of turbocharger engines and aircraft, combined. Therefore, it produces a very powerful stream of air flow. 


\section{IJIREEICE}

\section{International Journal of Innovative Research in \\ Electrical, Electronics, Instrumentation and Control Engineering}

Vol. 9, Issue 10, October 2021

DOI: 10.17148/IJIREEICE.2021.91015

Acceleration

As the air continues to flow in, it is accelerated by the open opening. Passing over a $16^{\circ}$ air-shaped ramp, the air is transferred to an empty ring.

Encouragement

The air flows inside the empty ring in a circular motion and is pushed through $16 \mathrm{~mm}$ thin lines.

\section{Power}

Traditional Drones with movers are dangerous because they can cause cuts to humans and birds. Also, if drones do not accidentally hit an obstacle, critical propellers can easily be injured. This concept of Propeller-less Drone removes such hazards and uses Dyson Bladeless Fan technology to not only be dangerous but also sustainable, as you need less than $40 \%$ energy, you can have 15 times more energy and use $45 \%$ more stable body products.

\section{Admission}

The surrounding air is absorbed quickly and this process is known as infiltration. The resulting air flow is stronger than a standard fan as it is amplified up to 15 times.

\section{Cooling bladeless fan}

The good thing about spotless fans is that they are very quiet. Destroying the air technology, these fans are absorbing more air and amplifying it on a larger scale. So, you can imagine how admirable these fans were in cooling off. Not only do they consume less energy, but they are also more powerful machines with more travel and safety features. They are designed to minimize turbulence in the airflow in an orderly fashion, thus providing a powerful and smooth airplane.

Impeller

An impeller is a rotating centrifugal pump that speeds fluid out from the center of rotation, thus transferring energy from the motor that carries the pump to the pumping fluid. The velocity obtained by the impeller transmits to the pressure where the external movement of the liquid is blocked by the pump cone. The impeller is usually a short cylinder with an opening (called eye) to receive incoming fluids, vans to press the fluid radially, and a separate, key, or corded vent to accommodate the drive shaft.

It would be less expensive to throw the impeller and its nail as a single piece, rather than separately. This combination is sometimes called "Rotor."

\section{Impeller Shaft}

The impeller is mounted on a shaft. Shaft is a mechanical component for transmitting torque from the motor to the impeller.

\section{PRINCIPLE AND WORKING}

\section{Coanda effect}

The Coanda effect describes the tendency of a jet to follow the contours of an adjacent boundary even when this boundary curves away from the initial jet axis. This effect either arises due to a) the pressure gradient perpendicular to a curved streamline, or b) differential entrainment and the development of a partial vacuum.

In the first case it can be shown using the Eiuler equation (or Bernoulli Equation in indistinct fluid) that the curved distribution tube meets the strength of the net facing the center of the curvature. Since there is no part of the viscous energy acting differently from the distribution tube, this means P1> P2. It is this pressure gradient that causes the jet to be removed from its original axis, and follows a result called Coanda.
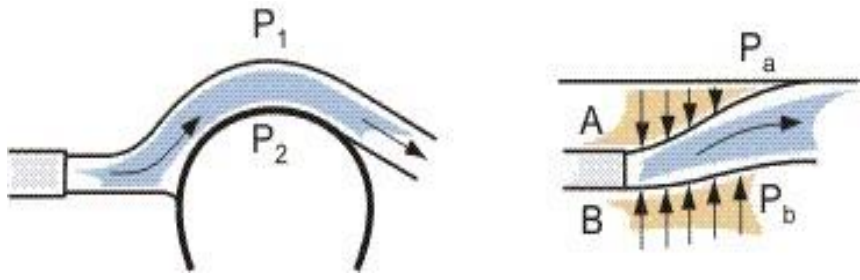

Fig. 3. Coanda effect

The free air jet incorporates air molecules from their surrounding areas causing axisymmetrical "tube" or "sleeve" of low pressure around the jet. Subsequent forces from this low-pressure tube eventually balance any circular flow instability, which stabilizes the jet in a straight line. However, if the solid area is placed close to each other, and closely resembles a jet, then the inflow (therefore removal) of air between the solid jet and the jet causes a decrease in air pressure on that side of the jet balance as quickly as the low pressure side on the "open" side of the jet. The pressure difference in the jet causes the plane to deviate in a straight line, and then stick to it. 


\section{IJIREEICE}

\section{International Journal of Innovative Research in \\ Electrical, Electronics, Instrumentation and Control Engineering}

Vol. 9, Issue 10, October 2021

\section{DOI: 10.17148/IJIREEICE.2021.91015}

The jet adheres even better to other curved areas, because each change (very small) that adds to the surface alignment brings the effects described by the initial bending of the jet to the surface. If the surface is not slightly curved, the plane, under ideal conditions, can stick to the surface even after a $180^{\circ}$ flow around the curved cylinder area, and thus move in the opposite direction to its original direction. The force that causes these changes in the direction of the jet flow causes equal and contradictory forces on the surface where the jet flows. These coanda-influenced festivals can be tied to cause heights and other forms of movement, depending on the shape of the jet and the surface the jet is attached to. A small "lip" on the surface where the plane begins to flow over that area develops the first deviation of the direction of flow of the jet, and then adheres to the surface.

\section{Working of bladeless drone}

- Impeller powered by BLDC motor sucks in air vertically and transmits the air to horizontal shaft at constant velocity.

- $\quad$ Electronic flow control valves are used to regulate the airflow to help change the drone speed and direction.

- Helmholtz cavity in the body reduces the noise of the air suction in the impeller.

- $\quad$ Annoying sound frequencies around $1000 \mathrm{~Hz}$ are eliminated

- $\quad$ The high velocity air is divided equally among the 4 shafts and then it flows around the loops after passing through the valve.

- $\quad$ Flow of air in dyson fan

- The air flows out of the loop through the $2 \mathrm{~mm}$ slot. It sticks to the outward concave slope of the loop body due to coanda effect.

A low-pressure vortex forms behind the lip, promoting the dip of the jet towards the surface. The Coanda effect can be induced in any fluid, and is therefore equally effective in water as in air.

- Low pressure - low pressure is created due to the air from loop flowing outwards.

- High pressure - high pressure exists above the loop, causing air to flow through the loop from high to low pressure

- $\quad$ This effect is called inducement of air, resulting in 15 times more than air flow rate than a same sized propeller.

- A vortex is formed due to the turbulent flow of the air which exists the loop.

- This vortex results in low pressure area creation, which attracts more air downwards.

- $\quad$ This effect is called entrainment of air.

The developers are the final details of the drones icon in our shared knowledge, and this is also how they achieve their vertical and flying navigation skills.

One could also say that it is the most important part of the drone. And it is very dangerous. In some cases, the drone ends up blazing without direction, disobeying your flight instructions. To combat this problem, some manufacturers have installed security guards using propellers. Some of the most advanced models come with anti-collision systems and collision technology. All of this helps to keep the drone propellers safe. And they work with varying degrees of success.

Can we fly without air?

Centrifugal fans are the kind you can find in leaf blowers and hair dryers. They breathe in the back. But instead of pushing the front out like a regular fan, they let the air out. The centrifugal fans on the Impeller Drone are arranged in such a way that they look like wheels on a car.The air is drawn to the sides, then pushed down. The design of the cover around the fans makes this possible by directing the flow of air where it should go.

The cover also protects the impeller from contact with anything on the outside, protecting the built-in air intake. The benefits of this impeller set are not hard to see. Your drone is very safe from collisions and irreparable damage to important objects. The protective houses around the breeze also allow air to be pushed harder through the exit area. This means that the drone flies high with low power. The only bad thing that no one can escape, however, is the noise that will be made when the drone is operating. It's the same situation as when you turn on your blower blower, so there's no surprise there.

\section{The role of production}

The manufacturing process is designed to ensure that the highest quality propellers are produced with improved drone performance during flight. Equality and quality control is enforced through the use of injection mold. Each plane must be fully aligned with the others, and powerful enough to operate freely. This is important for high performance during flight. Vibration control is smooth during production process to ensure minimal vibration when the drone is operating. This ensures that the image and video quality is not compromised during flight. The importance of proper production really shines through when a drone has to fly during inclement weather. For example, some drones are designed for solid tasks such as chasing storms and other natural weather conditions, providing live coverage. 
International Journal of Innovative Research in Electrical, Electronics, Instrumentation and Control Engineering

Vol. 9, Issue 10, October 2021

DOI: 10.17148/IJIREEICE.2021.91015

5.DIMENSIONS

Impeller

- $\quad$ Impeller width (mm)

b 50

- Impeller eye diameter(mm)

d 30

- No of vanes

z 4 Vane outlet angle

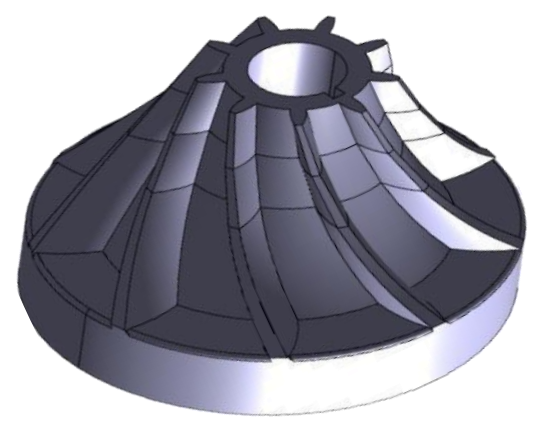

Fig. 4. Impeller

\section{Dyson fan}

- Dyson fan (inner)

$5 \mathrm{~cm}$

$7 \mathrm{~cm}$

$1 \mathrm{~cm}$

$\begin{array}{ll}\text { - } & \text { Thickness } \\ \text { - Cylindrical hollow }\end{array}$

4. $5 \mathrm{~cm}$

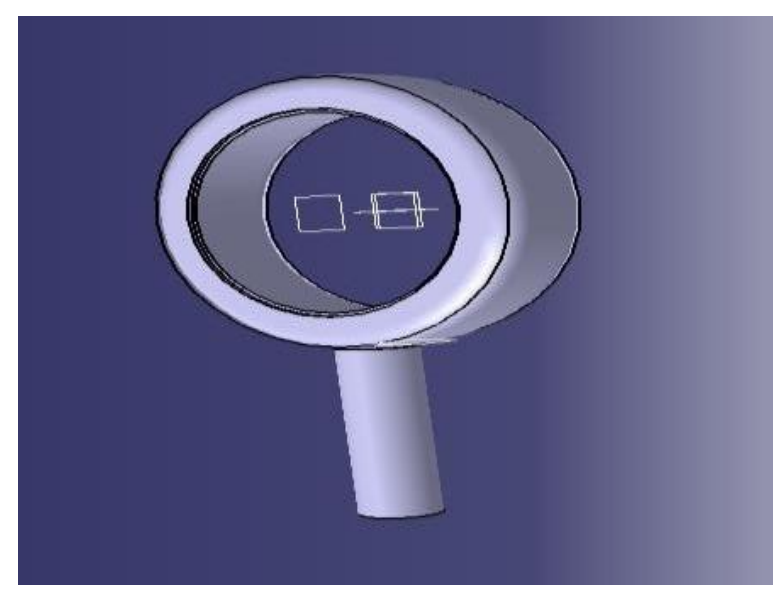

Fig. 5. Design of Dyson fan

Impeller shaft

Length

Diameter (d1)

Diameter (d2)

$300 \mathrm{~mm}$

$9.5 \mathrm{~mm}$

$8.5 \mathrm{~mm}$

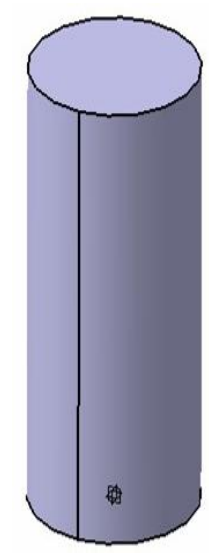

Fig. 6. Design of Impeller shaft 


\section{International Journal of Innovative Research in Electrical, Electronics, Instrumentation and Control Engineering}

Vol. 9, Issue 10, October 2021

\section{DOI: 10.17148/IJIREEICE.2021.91015}

\section{THE CONCLUSION}

The non-bladeless propulsion system is one of the most advanced carriers of UAVs with multiple rotors. Thrusters are designed to avoid the dangers posed by a helicopter to human life, property and the environment. Although this design does not provide enough for the aircraft but it has been proven that the pitch roll and yaw can be controlled. Initially the yaw head was impossible to control due to the gyroscopic effect performed. The gyroscopic effect, which refers to uncontrolled manipulation when the loss of control occurs, is most likely due to a combination of multiple complications. The first was due to the inertia times of each vehicle operating on different planes. Second is that the ball in the tripod socket was not directly in the center of the weight of the platform. To solve this problem the inclination of thrusters has been used to counteract the gyroscopic effect.

\section{REFERENCES}

1. Design and Development of a Bladeless Thruster for Autonomous Multicopters. Daniel Valdenegro1, Austin Capunay1, Daniel Gonzalez1, Luis Rodolfo Garcia Carrillo2 Member, IEEE, and Pablo Rangel3 2018.

2. 2.B. Rasuo and N. Mirkov, "On the possibility of using Coanda Effect for Unmanned Aerial Vehicles a numerical investigation", Proceedings of Applied Math. Mech., vol. 14, issue 1, pp. 627.628, 2014.

3. Propeller-Less Drone Concept Harmless, Energy Saving and Sustainable Innovation Nilesh Gandhi-2020

4. Propeller-less multi-rotor drone Ignacio Aguirre Panadero Director: Luke A. Wendt

(University of Illinois at Urbana-Champaign) 2016

5. Going Bladeless By Renee Knight Air, Ius Exclusive October 23, 2018

6. Bladeless Propulsion System Developed To Replace Propellers John Mohyi Ansys Blog September 23, 2017

7. How the dyson fan works - Jonathan Strickland and Nathan chandler - 2009

8. Red Dot - https://www.red-dot.org/project/bladeless-drone-26825 - Dr.Peter zec -2016 